\title{
A DYNAMIC STRATEGY FOR LARGE DATA REPLICATION IN HETEROGENEOUS NETWORK TOPOLOGIES
}

\author{
Cristian-Sorin BOLOGA \\ Business Informatics Research Center, Babes-Bolyai University, Cluj-Napoca, Romania \\ cristian.bologa@econ.ubbcluj.ro \\ Alexandru-Ioan STAN \\ Business Informatics Research Center, Babes-Bolyai University, Cluj-Napoca, Romania \\ alexandru.stan@econ.ubbcluj.ro \\ Doru-Catalin PASCA \\ doruc.pasca@gmail.com
}

\begin{abstract}
In this paper, we propose an efficient method for replicating large data volumes across servers and clusters (endpoints) having geographically heterogeneous locations. The endpoints may be placed in countries with different connectivity environments while the data is as large as 5 to 10 Terabytes. Our solution relies upon a dynamic algorithm which finds the optimal paths for the data replication process from the initial node to the rest of the endpoints. In the diffusion network the edges are valued so as to maximize the utility of stable connections possessing low latency and high speed. The dynamic assessment of utilities asserts our solutions' effectiveness in topologies undergoing frequently changing connectivity conditions.
\end{abstract}

Keywords: large data replication, optimization strategy, distributed systems.

JEL classification: C61, C63

DOI: $10.12948 / \mathrm{ie} 2019.02 .02$

\section{Introduction}

Nowadays, enormous volumes of data are produced in all activity fields and distributed storage strategies allow organizations to share it around the world [1-5]. The access to large data volumes generally becomes slower and more difficult as the number of nodes in the topology multiply.

In this context, large volume data replication emerge as a subject of paramount importance when advancing data availability in long-distance network topologies. In such heterogeneous environments, data replication is required for users and applications to retrieve and process the requested data from storages residing in neighbouring nodes [6-8]. To this purpose, large scale systems may generate large or even huge amount of replicas in geographically distributed locations, so that users can access them.

In this paper, we approach large volume data replication as an optimization technique that tries to increase the number of replica nodes and minimize the replication time. Our solution relies upon a dynamic algorithm which finds the optimal paths for data replication process from the initial source node to the rest of the endpoints.

This paper is organized as follows: section 2 is devoted to the empirical strategy chosen in this research. The deployment environment, the data replication algorithm and the experimental plan are also detailed. Results and conclusions are presented in section 3 and 4. 
www.conferenceie.ase.ro

\section{Replication strategy}

We propose an efficient practical method for replicating large data volumes across servers and clusters having geographically heterogeneous locations. The endpoints (servers or clusters) may be placed in countries with different connectivity environments such as Romania, US, Island, Germany, China or South Korea, while the data can be as large as 5 to 10 Terabytes. Each country generally has a few dozens of endpoints.

Our objective is to replicate the data in an optimal manner from a starting point, located in our case in Romania, to the rest of the endpoints through a shortest paths algorithm. To optimize the usage of network connections we associate them different utility functions. In the data diffusion network the edges have utilities which are maximized by stable connections possessing low the latency and high speed.

In essence, the transfer of data follows a high level country topology as in the picture below, with a starting node appearing in Romania. Our dynamic routing protocol relies upon the Dijkstra algorithm to find the shortest path between nodes and make the communication fast and easy.



Fig. 1. A schematic country-level perspective of our data deployment context

\subsection{A description of the deployment context}

We tested our data replication algorithm in the following environment. The servers are distributed across 6 countries: Island, Germany, Romania, Russia, United States and China. Each country has different external connectivity conditions. For instance, the external upload speed for China is about $20 \mathrm{Mbps}$. This means that a terabyte of data can be uploaded in just above five days. Connection latencies between the endpoints in the network play an important role too. For instance, current values for the connection latency between Romania and China are between 250 and 300 milliseconds. As for the physical layer, the connections between the sub topologies of each country were of the type uplink 10G base T.

The effective replication of the data was done using the rsync and the lsyncd commands as all the servers were running Linux operating systems.

For instance, to copy a folder to a server in Germany we run the rsynch command with the following parameters: rsync -avzu /data 192.168.1.104:/data. For better results we also used rsynch, instead of lsyncd. The main difference between the commands is that the former has a dedicated service monitoring in real-time the changes in the data content which readily pushes the deltas towards the destination servers. 
www.conferenceie.ase.ro

Our solution had to cope with four types of connectivity problems:

- Connection latencies greater than $1000 \mathrm{~ms}$, in which case the connections are soon be lost and new alternative routes should be found;

- Connection Latencies greater than $700 \mathrm{~ms}$, often implying subsequent package delays and network congestion if large amounts of packages are rerouted;

- Connectivity problems related to the administrative and legislative regulations of the host country (package filtering in China which slows down cross-border internet traffic and regulate the Internet domestically; Russia also plans to tightly regulate the external connections)

- Very slow connections resulting from the poor infrastructure of a country/region.

The connections with the countries posing regulatory obstacles were done either through virtual private networks (VPNs) or, in China's case, by using a MPLS (Multiprotocol Label Switching). MPLS is a routing network mechanism which directs data among different nodes belonging potentially to the same company's infrastructure. We used it because it enables very stable and scalable connections, and facilitates the creation of virtual connections between faraway nodes.

The nodes in the network had either master or slave roles. The nodes were slaves as soon as they start getting the data but the replication process was not completed. Masters were nodes having completed the data transfer. In the figure below, we see an example of master slave topology using 10G Base T connections.

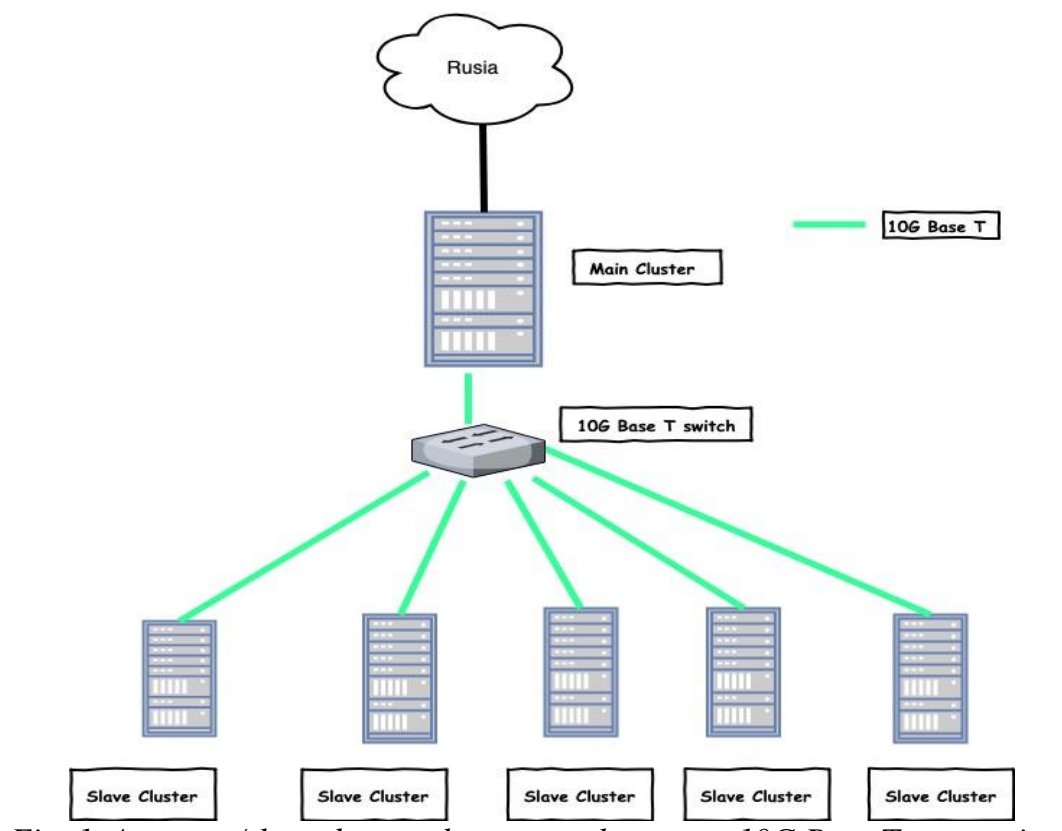

Fig. 1. A master/slave data exchange topology over 10G Base T connections

\subsection{Adapting the Dijkstra Algorithm}

To implement the data replication algorithm we have to find the time costs needed to transfer data from our initial node in Romania to the rest of the nodes in the graph. The main cost parameters are obtained by: 
- Pinging the IP of each destination node;

- Sending out a set of small-sized files and using the empirical average of the observed latencies and upload speeds.

After getting the main cost parameters we compute the adjacency matrix using different utility functions.

Table 1. The adjacency matrix for the replication algorithm

\begin{tabular}{|l|c|c|c|c|c|c|}
\hline Countries(hosts) & Island & Germany & Romania & Russia & U.S. & China \\
\hline Island & 0 & $\mathrm{U}(45, \mathrm{st})$ & 0 & 0 & 0 & $\mathrm{U}(320, \mathrm{st})$ \\
\hline Germany & $\mathrm{U}(45, \mathrm{st})$ & 0 & $\mathrm{U}(30, \mathrm{st})$ & 0 & 0 & $\mathrm{U}(210, \mathrm{st})$ \\
\hline Romania & 0 & $\mathrm{U}(30, \mathrm{st})$ & 0 & $\mathrm{U}(190, \mathrm{st})$ & $\mathrm{U}(330, \mathrm{st})$ & 0 \\
\hline Russia & 0 & 0 & $\mathrm{U}(190, \mathrm{st})$ & 0 & $\mathrm{U}(10, \mathrm{st})$ & 0 \\
\hline U.S. & 0 & 0 & $\mathrm{U}(330, \mathrm{st})$ & $\mathrm{U}(10, \mathrm{st})$ & 0 & $\mathrm{U}(280, \mathrm{st})$ \\
\hline China & $\mathrm{U}(320, \mathrm{st})$ & $\mathrm{U}(210, \mathrm{st})$ & 0 & 0 & $\mathrm{U}(280, \mathrm{st})$ & 0 \\
\hline
\end{tabular}

The network contains specific sub-graphs for each country. Each country sub-graph has a predefined starting point which can be back-upped when it becomes unavailable. We apply the algorithm on each sub-graph at regular time intervals. When the file transfer starts off onto a destination server, that node is temporarily excluded from the graph.

\section{Results}

The volume of data we used in our tests were ranging between 1 and 20 Terabytes. We repeated the deployment process for a few dozens of times for most of the data volumes. We only took in consideration the deployments completed successfully as we encountered moderate failure rates. We chose to send large volumes of small-granularity data files (mainly large sets of rather small-sized pictures).

Table 2. The mean replication times and their standard deviations (SDs) for the two benchmarks as compared to those induced by our strategy $(100 \%)$

\begin{tabular}{|l|c|c|c|c|c|c|}
\hline Data volume & $\mathbf{1 ~ T B}$ & $\mathbf{5 ~ T B}$ & $\mathbf{7 ~ T B}$ & $\mathbf{1 0}$ TB & $\mathbf{1 5}$ TB & $\mathbf{2 0}$ TB \\
\hline $\begin{array}{l}\text { Erdős-Rényi mean } \\
\text { replication time }\end{array}$ & $223 \%$ & $226 \%$ & $232 \%$ & $237 \%$ & $538 \%$ & $764 \%$ \\
\hline $\begin{array}{l}\text { Barabási-Albert mean } \\
\text { replication time }\end{array}$ & $112 \%$ & $112 \%$ & $112 \%$ & $119 \%$ & $187 \%$ & $280 \%$ \\
\hline $\begin{array}{l}\text { Erdős-Rényi replication } \\
\text { time SD }\end{array}$ & $2314 \%$ & $2443 \%$ & $2697 \%$ & $2788 \%$ & $3131 \%$ & $5538 \%$ \\
\hline $\begin{array}{l}\text { Barabási-Albert } \\
\text { replication time SD }\end{array}$ & $354 \%$ & $357 \%$ & $421 \%$ & $698 \%$ & $1103 \%$ & $1672 \%$ \\
\hline
\end{tabular}

We tested the effectiveness of our deployment strategy by comparing it with two replication benchmarks. A first benchmark was a diffusion process in an Erdős-Rényi[9] random replication network generated over our topology. A second benchmark was a Barabási-Albert [10] preferential attachment model in which the more connected nodes are more likely to be 
used as data dispatchers. To assess the speed and the completeness of the deployment process all the endpoints sent back deployment reports to a collecting server.

We obtained deployment times two to three times better by using our algorithm as compared to the mean time in the case of typical Erdős-Rényi random replication network. Also, the stability of our solution was higher with smaller standard deviations in overall replication timespans. When compared to the average replication time using the Barabási-Albert topology our solution worked slightly better, perhaps 15 to 20 percent faster.

Table 3. The mean replication times induced by our strategy for different data volumes and the percentages of critical nodes having large transmission loads

\begin{tabular}{|l|c|c|c|c|c|c|}
\hline Data volume & 1 TB & 5 TB & 7 TB & 10 TB & 15 TB & 20 TB \\
\hline $\begin{array}{l}\text { Mean replication time } \\
\text { (our strategy) }\end{array}$ & $100 \%$ & $471 \%$ & $694 \%$ & $1080 \%$ & $2316 \%$ & $572 \%$ \\
\hline $\begin{array}{l}\text { Critical nodes usage } \\
\text { (our strategy) }\end{array}$ & $93.94 \%$ & $95.52 \%$ & $95.53 \%$ & $89.18 \%$ & $81.29 \%$ & $73.90 \%$ \\
\hline $\begin{array}{l}\text { critical nodes usage } \\
\text { (Erdős-Rényi) }\end{array}$ & $28.3 \%$ & $36.41 \%$ & $30.86 \%$ & $24.5 \%$ & $27.88 \%$ & $29.41 \%$ \\
\hline $\begin{array}{l}\text { critical nodes usage } \\
\text { (Barabási-Albert) }\end{array}$ & $90.71 \%$ & $90.34 \%$ & $92.71 \%$ & $91.12 \%$ & $87.22 \%$ & $88.3 \%$ \\
\hline
\end{tabular}

The solution also offers good scalability with replication times linear in data volumes up to 10 terabytes: the replication of a terabyte of data is about 10 times shorter than the replication of 10 terabytes of data. Beyond $10 \mathrm{~TB}$ of data the performances decrease moderately but the failure rates are significantly higher. The dynamic nature of our strategy made it very resilient in the case of fast changing connectivity conditions. Our replication strategy also makes better use of the network links having a more critical role in propagating information by putting higher transmission loads on them. Generally, these links had higher probability in partaking in the replication process when compared to the benchmarks.

\section{Conclusions}

In this article we gauge the effectiveness of a data replication strategy over large volumes of data in a geographically heterogeneous network topology. The nodes in the network are placed in countries with different connectivity environments while the data can be as large as 5 to 10 Terabytes. Our algorithm dynamically finds the optimal paths for the replication process from the initial node to the rest of the endpoints. In the data diffusion network the edges are valued so as to maximize the utility of stable connections possessing low latency and high transfer speed. Our strategy is faster and more stable than random diffusion benchmark processes over Erdős-Rényi and Barabási-Albert topologies with smaller standard deviations in replication timespans. The dynamic assessment of utilities asserts our solution' effectiveness in topologies undergoing fast changing connectivity conditions. The solution also offers good scalability with replication times linear in data volumes. 
www.conferenceie.ase.ro

\section{References}

[1] H. Lamehamedi, B. Szymanski, Z. Shentu, and E. Deelman, Data replication strategies in grid environments, in Proc. of the 5th IEEE International Conference on Algorithms and architectures for Parallel Processing, Beijing, China, 2002, pp. 378-383.

[2] A. Nahir, A. Orda, and D. Raz, Replication-based load balancing, IEEE Transactions on Parallel and Distributed Systems 27 (2016), pp. 494-507.

[3] S.M. Park, J.H. Kim, Y.B. Ko, and W.S. Yoon, Dynamic data grid replication strategy based on internet hierarchy, in Grid and Cooperative Computing(GCC 2003), Lecture Notes in Computer Science, Vol. 3033, Springer, Berlin, Heidelberg, 2004, pp. 838-846.

[4] S. Souravlas, and A. Sifaleras, Trends in data replication strategies: A survey. International Journal of Parallel Emergent and Distributed Systems. 34. (2019) , pp 222239. 10.1080/17445760.2017.1401073.

[5] W. Li, Y. Yang, and D. Yuan, Ensuring cloud data reliability with minimum replication by proactive replica checking, IEEE Transactions on Computers 65 (2016), pp. 14941506.

[6] R. Souli-Jbali, M.S. Hidri, and R.B. Ayed, Dynamic data replication-driven model in data grids, in Proc. of the 39th IEEE Annual Computer Software and Applications Conference (COMPSAC), 1-5 July, Taichung, Taiwan, 2015, pp.393-397.

[7] G.A. Michelon, L.A. Lima, J.A. Oliveirade, A. Calsavara, and G.E. Andradede , A strategy for data replication in mobile ad hoc networks, in Proc. of the 22nd IEEE International Symposium on Modelling, Analysis \& Simulation of Computer and Telecommunication Systems (MASCOTS), 9-11 September, Paris, France, 2014, pp. 486-489.

[8] E. Spaho, L. Barolli, and F. Xhafa, Data replication strategies in P2P systems: a survey, in Proc. of the 17th IEEE International Conference on Network-Based Information Systems (NBiS), 10-12 September, Salerno, Italy, 2014,pp. 302-309.

[9] P. Erdős, and A.Rényi, On the evolution of random graphs. Publications of the Mathematical Institute of the Hungarian Academy of Sciences (1960).

[10] A. Barabási, and R. Albert, Emergence of scaling in random networks, Science. 286 (5439), 1999, pp. 509-512. 